\title{
ON CONNECTING WEYL-ORBIT FUNCTIONS TO JACOBI POLYNOMIALS AND MULTIVARIATE (ANTI)SYMMETRIC TRIGONOMETRIC FUNCTIONS
}

\author{
JiŘí HrivnÁK, LEnKa MotlochovÁ*
}

\author{
Department of Physics, Faculty of Nuclear Sciences and Physical Engineering, Czech Technical University in \\ Prague, Břehová 7, CZ-115 19 Prague, Czech Republic \\ * corresponding author: lenka.motlochova@fjfi.cvut.cz
}

\begin{abstract}
The aim of this paper is to make an explicit link between the Weyl-orbit functions and the corresponding polynomials, on the one hand, and to several other families of special functions and orthogonal polynomials on the other. The cornerstone is the connection that is made between the one-variable orbit functions of $A_{1}$ and the four kinds of Chebyshev polynomials. It is shown that there exists a similar connection for the two-variable orbit functions of $A_{2}$ and a specific version of two variable Jacobi polynomials. The connection with recently studied $G_{2}$-polynomials is established. Formulas for connection between the four types of orbit functions of $B_{n}$ or $C_{n}$ and the (anti)symmetric multivariate cosine and sine functions are explicitly derived.
\end{abstract}

KEYwords: Weyl-orbit functions, Chebyshev polynomials, Jacobi polynomials, (anti)symmetric trigonometric functions.

\section{INTRODUCTION}

Special functions associated with the root systems of simple Lie algebras, e.g. Weyl-orbit functions, play an important role in several domains of mathematics and theoretical physics, in particular in representation theory, harmonic analysis, numerical integration and conformal field theory. The purpose of this paper is to link Weyl-orbit functions with various types of orthogonal polynomials, namely Chebyshev and Jacobi polynomials and their multivariate generalizations, and thus to motivate further development of the remarkable properties of these polynomials in connection with orbit functions.

The collection of Weyl-orbit functions includes four different families of functions called $C^{-}, S_{-}, S^{s_{-}}$and $S^{l}-$ functions [6, 15, 16, 25. They are induced from the sign homomorphisms of the Weyl groups of geometric symmetries related to the underlying Lie algebras. The symmetric $C$-functions and antisymmetric $S$ functions also appear in the representation theory of simple Lie algebras [32, 34]; the $S$-functions appear in the Weyl character formula and every character of irreducible representations of simple Lie algebra can be written as a linear combination of $C$-functions. Unlike $C$ - and $S$-functions, $S^{s_{-}}$and $S^{l}$-functions exist only in the case of simple Lie algebras with two different lengths of roots.

A review of several pertinent properties of the Weylorbit functions is contained in [8, 15, 16, 25]. These functions possess symmetries with respect to the affine Weyl group - an infinite extension of the Weyl group by translations in dual root lattice. Therefore, we consider $C_{-}, S_{-}, S^{s_{-}}$and $S^{l}$-functions only on specific subsets of the fundamental domain $F$ of the affine
Weyl group. Within each family, the functions are continuously orthogonal when integrated over $F$ and form a Hilbert basis of squared integrable functions on $F[25,27]$. They also satisfy discrete orthogonality relations which is of major importance for the processing of multidimensional digital data $8,10,27$. Using discrete Fourier-like transforms arising from discrete orthogonality, digital data are interpolated in any dimension and for any lattice symmetry afforded by the underlying simple Lie algebra. Several special cases of simple Lie algebras of rank two are studied in 28 30].

The properties of orbit functions also lead to numerical integration formulas for functions of several variables. They approximate a weighted integral of any function of several variables by a linear combination of function values at points called nodes. In general, such formulas are required to be exact for all polynomial functions up to a certain degree [3]. Furthermore, the $C$-functions and $S$-functions of simple Lie algebra $A_{1}$ coincide, up to a constant, with the common cosine and sine functions respectively. They, are therefore, related to the extensively studied Chebyshev polynomials and, consequently, to the integration formulas, quadratures, for the functions of one variable [5, 31. In [24, it is shown that there are analogous formulas for numerical integration, for multivariate functions, that depend on the Weyl group of the simple Lie algebra $A_{n}$ and the corresponding $C$ - and $S$-functions. The resulting rules for functions of several variables are known as cubature formulas. The idea of 24 is extended to any simple Lie algebra in $9,25,26$. Optimal cubature formulas in the sense of the nodal points that are required are known only for $S$ - and $S^{s}$-functions. 
Besides the Chebyshev polynomials, the Weyl-orbit functions are related to other orthogonal polynomials. For example, orbit functions of $A_{2}$ and $C_{2}$ coincide with two-variable analogues of Jacobi polynomials 21]. It can also be shown that the $C_{-}, S-$, $S^{s}$ - and $S^{l}$-functions arising in connection with simple Lie algebras $B_{n}$ and $C_{n}$ become, up to a constant, (anti)symmetric multivariate cosine functions and (anti)symmetric multivariate sine functions [17. Note that these generalizations lead to multivariate analogues of Chebyshev polynomials and are used to derive optimal cubature formulas. Therefore, this fact indicates that it might be possible to obtain such formulas for all families of orbit functions. As for Chebyshev polynomials, it is of interest to study the accuracy of approximations and interpolations using cubature formulas.

This paper starts with a brief introduction to Weyl groups in Section 2. Then there is a review of the relations of the Weyl-orbit functions with other special functions which are associated with the Weyl groups. In Section 3.1 the connection of the $C$ - and $S$-functions of one variable with Chebyshev polynomials is recalled. In Sections 3.2 and 3.4 , we show that each family of Weyl-orbit functions corresponding to $A_{2}$ and $C_{2}$ can be viewed as a two-variable analogue of Jacobi polynomials [21. In Section 3.4. we also provide the exact connection with generalizations of trigonometric functions [34.

\section{WEYL GROUPS OF SIMPLE LIE ALGEBRAS}

In this section, we summarize the properties of the Weyl groups that are neeeded for definition of orbit functions. There are four series of simple Lie algebras $A_{n}(n \geq 1), B_{n}(n \geq 3), C_{n}(n \geq 2), D_{n}(n \geq 4)$ and five exceptional simple Lie algebras $E_{6}, E_{7}, E_{8}, F_{4}$ and $G_{2}$. Each of these algebras is connected with its corresponding Weyl group [1, 12, 13, 18, 34]. They are completely classified by Dynkin diagrams (see e.g. figure in [15]). A Dynkin diagram characterizes a set $\Delta$ of simple roots $\alpha_{1}, \ldots, \alpha_{n}$ generating an Euclidean space isomorphic to $\mathbb{R}^{n}$ with the scalar product denoted by $\langle\cdot, \cdot\rangle$. Each node of the Dynkin diagram represents one simple root $\alpha_{i}$. The number of links between two nodes corresponding to $\alpha_{i}$ and $\alpha_{j}$ respectively is equal to

$$
\left\langle\alpha_{i}, \alpha_{j}^{\vee}\right\rangle\left\langle\alpha_{j}, \alpha_{i}^{\vee}\right\rangle, \quad \text { where } \alpha_{i}^{\vee} \equiv \frac{2 \alpha_{i}}{\left\langle\alpha_{i}, \alpha_{i}\right\rangle} .
$$

Note that we use the standard normalization for the lengths of roots, namely $\left\langle\alpha_{i}, \alpha_{i}\right\rangle=2$ if $\alpha_{i}$ is a long simple root.

In addition to the basis of $\mathbb{R}^{n}$ consisting of the simple roots $\alpha_{i}$, it is convenient for our purposes to introduce the basis of fundamental weights $\omega_{j}$ given by

$$
\left\langle\omega_{j}, \alpha_{i}^{\vee}\right\rangle=\delta_{i j}
$$

This allows us to express the weight lattice $P$ defined by

$$
P \equiv\left\{\lambda \in \mathbb{R}^{n} \mid\left\langle\lambda, \alpha_{i}^{\vee}\right\rangle \in \mathbb{Z}, i=1, \ldots, n\right\}
$$

as $\mathbb{Z}$-linear combinations of $\omega_{j}$. The subset of dominant weights $P^{+}$is standardly given as

$$
P^{+} \equiv \mathbb{Z}^{\geq 0} \omega_{1}+\cdots+\mathbb{Z}^{\geq 0} \omega_{n}
$$

We consider the usual partial ordering on $P$ given by $\mu \preceq \lambda$ if and only if $\lambda-\mu$ is a sum of simple roots with non-negative integer coefficients.

To each simple root $\alpha_{i}$ there is a corresponding reflection $r_{i}$ with respect to the hyperplane orthogonal to $\alpha_{i}$

$$
r_{i}(a) \equiv r_{\alpha_{i}}(a)=a-\frac{2\left\langle a, \alpha_{i}\right\rangle}{\left\langle\alpha_{i}, \alpha_{i}\right\rangle} \alpha_{i}, \quad \text { for } a \in \mathbb{R}^{n} .
$$

The finite group $W$ generated by such reflections $r_{i}, i=1, \ldots, n$ is called the Weyl group. For the properties of the Weyl groups see e.g. [12, 14].

In the case of simple Lie algebras with two different lengths of the roots, we need to distinguish between short and long simple roots. Therefore, we denote by $\Delta_{s}$ the set of simple roots containing only short simple roots and we denote by $\Delta_{l}$ the set of long simple roots. We also define the following vectors:

$$
\varrho \equiv \sum_{i=1}^{n} \omega_{i}, \quad \varrho^{s} \equiv \sum_{\alpha_{i} \in \Delta_{s}} \omega_{i}, \quad \varrho^{l} \equiv \sum_{\alpha_{i} \in \Delta_{l}} \omega_{i} .
$$

\section{WEYL-ORBIT FUNCTIONS}

Each type of Weyl-orbit function arises from the sign homomorphism of Weyl groups $\sigma: W \rightarrow\{ \pm 1\}$. There exist only two different sign homomorphisms on $W$ connected to simple Lie algebras with one length of the roots: identity, denoted by 1 , and the determinant 8 , 25]. They are given by their values on the generators $r_{i}$ of $W$ as

$$
\begin{array}{cc}
\mathbf{l}\left(r_{i}\right)=1 & \text { for all } \alpha_{i} \in \Delta, \\
\operatorname{det}\left(r_{i}\right)=-1 & \text { for all } \alpha_{i} \in \Delta .
\end{array}
$$

In the case of simple Lie algebras with two different lengths of roots, i.e. $B_{n}, C_{n}, F_{4}$ and $G_{2}$, there are two additional sign homomorphisms denoted by $\sigma^{s}$ and $\sigma^{l}$ and given as

$$
\begin{gathered}
\sigma^{s}\left(r_{i}\right)= \begin{cases}-1 & \text { if } \alpha_{i} \in \Delta_{s}, \\
1 & \text { if } \alpha_{i} \in \Delta_{l},\end{cases} \\
\sigma^{l}\left(r_{i}\right)= \begin{cases}1 & \text { if } \alpha_{i} \in \Delta_{s}, \\
-1 & \text { if } \alpha_{i} \in \Delta_{l} .\end{cases}
\end{gathered}
$$

Labelled by the parameter $a \in \mathbb{R}^{n}$, the Weyl-orbit function of the variable $b \in \mathbb{R}^{n}$ corresponding to sign homomorphism $\sigma$ is introduced via the formula

$$
\varphi_{a}^{\sigma}(b)=\sum_{w \in W} \sigma(w) e^{2 \pi i\langle w(a), b\rangle}, \quad a, b \in \mathbb{R}^{n} .
$$


Each sign homomorphism 1 , det, $\sigma^{s}, \sigma^{l}$ determines one family of complex valued Weyl-orbit functions, called $C_{-}, S_{-}, S^{s_{-}}$and $S^{l-f u n c t i o n s}$ respectively, and denoted by

$$
\begin{array}{lll}
C \text {-functions: } & \sigma \equiv 1, & \varphi^{\sigma} \equiv \Phi, \\
S \text {-functions: } & \sigma \equiv \operatorname{det}, & \varphi^{\sigma} \equiv \varphi, \\
S^{s} \text {-functions: } & \sigma \equiv \sigma^{s}, & \varphi^{\sigma} \equiv \varphi^{s}, \\
S^{l} \text {-functions: } & \sigma \equiv \sigma^{l}, & \varphi^{\sigma} \equiv \varphi^{l} .
\end{array}
$$

Several remarkable properties of Weyl-orbit functions, such as continuous and discrete orthogonality are usually achieved by restricting $a$ to some subsets of the weight lattice $P$ (see for example [8, 10, 15, 16, 27]). Note also that symmetric $C$-functions and antisymmetric $S$-functions appear in the theory of irreducible representations of simple Lie algebras 32,34 .

It is also convenient to use an alternative definition of the Weyl-orbit functions via sums over the Weyl group orbits [15, 16, 25]. These orbit sums $C_{a}, S_{a+\varrho}$, $S_{a+\varrho^{s}}^{s}, S_{a+\varrho^{l}}^{l}$ differ from $\Phi_{a}, \varphi_{a+\varrho}, \varphi_{a+\varrho^{s}}^{s}, \varphi_{a+\varrho^{l}}^{l}$ only by a constant

$$
\begin{gathered}
C_{a}(b)=\frac{\Phi_{a}(b)}{\left|\operatorname{Stab}_{W} a\right|}, \quad S_{a+\varrho}(b)=\varphi_{a+\varrho}(b), \\
S_{a+\varrho^{s}}^{s}(b)=\frac{\varphi_{a+\varrho^{s}}^{s}(b)}{\left|\operatorname{Stab}_{W}\left(a+\varrho^{s}\right)\right|}, \\
S_{a+\varrho^{l}}^{l}(b)=\frac{\varphi_{a+\varrho^{l}}^{l}(b)}{\left|\operatorname{Stab}_{W}\left(a+\varrho^{l}\right)\right|}
\end{gathered}
$$

with $\left|\operatorname{Stab}_{W} c\right|$ denoting the number of elements of $W$ which leave $c$ invariant.

\subsection{CASE $A_{1}$}

The symmetric $C$-functions and antisymmetric $S$ functions of $A_{1}$ are, up to a constant, the common cosine and sine functions [15, 16,

$$
C_{a}(b)=2 \cos \left(2 \pi a_{1} b_{1}\right), \quad S_{a}(b)=2 i \sin \left(2 \pi a_{1} b_{1}\right),
$$

where $a=a_{1} \omega_{1}, b=b_{1} \alpha_{1}^{\vee}$. It is well known that such functions appear in the definition of the extensively studied Chebyshev polynomials [5, 31. Several types of Chebyshev polynomials are widely used in mathematical analysis, in particular, as efficient tools for numerical integration and approximations. The Chebyshev polynomials of the first, second, third and fourth kind are denoted by $T_{m}(x), U_{m}(x), V_{m}(x)$ and $W_{m}(x)$, respectively. If $x=\cos (\theta)$, then for any $m \in \mathbb{Z}^{\geq 0}$ it holds that

$$
\begin{aligned}
T_{m}(x) & \equiv \cos (m \theta), \\
U_{m}(x) & \equiv \frac{\sin ((m+1) \theta)}{\sin (\theta)}, \\
V_{m}(x) & \equiv \frac{\cos \left(\left(m+\frac{1}{2}\right) \theta\right)}{\cos \left(\frac{1}{2} \theta\right)}, \\
W_{m}(x) & \equiv \frac{\sin \left(\left(m+\frac{1}{2}\right) \theta\right)}{\sin \left(\frac{1}{2} \theta\right)} .
\end{aligned}
$$

Therefore, for specific choices of parameter $a_{1}$ and $2 \pi b_{1}=\theta$, we can view the Weyl-orbit functions of $A_{1}$ as these Chebyshev polynomials.

Recall also that the Chebyshev polynomials are actually, up to a constant $c_{\alpha, \beta}$, special cases of Jacobi polynomials $P_{m}^{(\alpha, \beta)}(x), m \in \mathbb{Z}^{\geq 0}$. The Jacobi polynomials are given as orthogonal polynomials with respect to the weight function

$$
(1-x)^{\alpha}(1+x)^{\beta}, \quad-1<x<1,
$$

where the parameters $\alpha, \beta$ are subjects to the condition $\alpha, \beta>-1$ [4, 33]. In particular, it holds that

$$
\begin{aligned}
& T_{m}(x)=c_{-\frac{1}{2},-\frac{1}{2}} P_{m}^{\left(-\frac{1}{2},-\frac{1}{2}\right)}(x), \\
& U_{m}(x)=c_{\frac{1}{2}, \frac{1}{2}} P_{m}^{\left(\frac{1}{2}, \frac{1}{2}\right)}(x), \\
& V_{m}(x)=c_{-\frac{1}{2}, \frac{1}{2}} P_{m}^{\left(-\frac{1}{2}, \frac{1}{2}\right)}(x), \\
& W_{m}(x)=c_{\frac{1}{2},-\frac{1}{2}} P_{m}^{\left(\frac{1}{2},-\frac{1}{2}\right)}(x) .
\end{aligned}
$$

For both Chebyshev polynomials and Jacobi polynomials, there exist various multivariate generalizations, see for example [3, 21, 22, In Sections 3.2, 3.3 and 3.4, we identify some of the two-variable analogous orthogonal polynomials with specific Weyl-orbit functions.

\subsection{CASE $A_{2}$}

Since, for $A_{2}$ the two simple roots are of the same length, there are only two corresponding families of Weyl-orbit functions, $C$ - and $S$-functions. For $a=$ $a_{1} \omega_{1}+a_{2} \omega_{2}$ and $b=b_{1} \alpha_{1}^{\vee}+b_{2} \alpha_{2}^{\vee}$ the explicit formulas of $C$-functions and $S$-functions are given by

$$
\begin{gathered}
C_{a}(b)=\frac{1}{\left|\operatorname{Stab}_{W} a\right|}\left(e^{2 \pi i\left(a_{1} b_{1}+a_{2} b_{2}\right)}\right. \\
+e^{2 \pi i\left(-a_{1} b_{1}+\left(a_{1}+a_{2}\right) b_{2}\right)}+e^{2 \pi i\left(\left(a_{1}+a_{2}\right) b_{1}-a_{2} b_{2}\right)} \\
+e^{2 \pi i\left(a_{2} b_{1}-\left(a_{1}+a_{2}\right) b_{2}\right)}+e^{2 \pi i\left(\left(-a_{1}-a_{2}\right) b_{1}+a_{1} b_{2}\right)} \\
\left.+e^{2 \pi i\left(-a_{2} b_{1}-a_{1} b_{2}\right)}\right), \\
S_{a}(b)=e^{2 \pi i\left(a_{1} b_{1}+a_{2} b_{2}\right)}-e^{2 \pi i\left(-a_{1} b_{1}+\left(a_{1}+a_{2}\right) b_{2}\right)} \\
-e^{2 \pi i\left(\left(a_{1}+a_{2}\right) b_{1}-a_{2} b_{2}\right)}+e^{2 \pi i\left(a_{2} b_{1}-\left(a_{1}+a_{2}\right) b_{2}\right)} \\
\quad+e^{2 \pi i\left(\left(-a_{1}-a_{2}\right) b_{1}+a_{1} b_{2}\right)}-e^{2 \pi i\left(-a_{2} b_{1}-a_{1} b_{2}\right)}
\end{gathered}
$$

with the values $\left|\operatorname{Stab}_{W} a\right|$ given in Table 1 of $[9]$.

They are related to the generalized cosine and sine functions $T C_{k}$ and $T S_{k}$ studied in [23] and defined as

$$
\begin{aligned}
T C_{k}(t)= & \frac{1}{3}\left(e^{\frac{i \pi}{3}\left(k_{2}-k_{3}\right)\left(t_{2}-t_{3}\right)} \cos k_{1} \pi t_{1}\right. \\
+ & e^{\frac{i \pi}{3}\left(k_{2}-k_{3}\right)\left(t_{3}-t_{1}\right)} \cos k_{1} \pi t_{2} \\
& \left.\quad+e^{\frac{i \pi}{3}\left(k_{2}-k_{3}\right)\left(t_{1}-t_{2}\right)} \cos k_{1} \pi t_{3}\right), \\
T S_{k}(t)= & \frac{1}{3}\left(e^{\frac{i \pi}{3}\left(k_{2}-k_{3}\right)\left(t_{2}-t_{3}\right)} \sin k_{1} \pi t_{1}\right. \\
+ & e^{\frac{i \pi}{3}\left(k_{2}-k_{3}\right)\left(t_{3}-t_{1}\right)} \sin k_{1} \pi t_{2} \\
& \left.\quad+e^{\frac{i \pi}{3}\left(k_{2}-k_{3}\right)\left(t_{1}-t_{2}\right)} \sin k_{1} \pi t_{3}\right),
\end{aligned}
$$




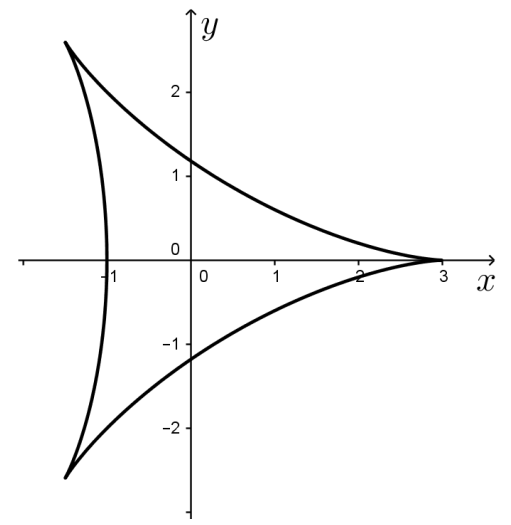

FiguRE 1. The region of orthogonality bounded by the three-cusped deltoid.

where $t=\left(t_{1}, t_{2}, t_{3}\right) \in \mathbb{R}^{3}$ with $t_{1}+t_{2}+t_{3}=0$ and $k=\left(k_{1}, k_{2}, k_{3}\right) \in \mathbb{Z}^{3}$ with $k_{1}+k_{2}+k_{3}=0$. The explicit correspondence

$$
C_{a}=\frac{6}{\left|\operatorname{Stab}_{W} a\right|} T C_{k}, \quad S_{a}=6 T S_{k}
$$

is obtained by the following change of variables and parameters,

$$
\begin{gathered}
k_{1}=a_{1}, \quad k_{2}=a_{2}, \quad k_{3}=-a_{1}-a_{2}, \\
t_{1}=2 b_{1}-b_{2}, \quad t_{2}=-b_{1}+2 b_{2}, \quad t_{3}=-b_{1}-b_{2} .
\end{gathered}
$$

It is possible to express $C_{a}$ and $S_{a+\varrho} / S_{\varrho}$ with $a \in$ $P^{+}$as polynomials in $C_{\omega_{1}}$ and $C_{\omega_{2}}$ [1]. Taking into account that $C_{\omega_{1}}=\overline{C_{\omega_{2}}}$, one can pass to real variables by making a natural change of variables, these

$$
\begin{array}{r}
x=\frac{C_{\omega_{1}}+C_{\omega_{2}}}{2}=\cos 2 \pi b_{1}+\cos 2 \pi b_{2} \\
+\cos 2 \pi\left(b_{1}-b_{2}\right), \\
y=\frac{C_{\omega_{1}}-C_{\omega_{2}}}{2 i}=\sin 2 \pi b_{1}-\sin 2 \pi b_{2} \\
-\sin 2 \pi\left(b_{1}-b_{2}\right) .
\end{array}
$$

Since $C$-functions and $S$-functions are continuously orthogonal, their polynomial versions inherit the orthogonality property. One can verify that the corresponding polynomials are special cases of twodimensional analogues of Jacobi polynomials orthogonal with respect to the weight function

$w_{\alpha}(x, y)=\left(-\left(x^{2}+y^{2}+9\right)^{2}+8\left(x^{3}-3 x y^{2}\right)+108\right)^{\alpha}$

on the region bounded by the three-cusped deltoid called Steiner's hypocycloid [21] with the boundary given by

$$
-\left(x^{2}+y^{2}+9\right)^{2}+8\left(x^{3}-3 x y^{2}\right)+108=0,
$$

see Fig. 1. More precisely, the polynomials $C_{a}$ and $S_{a+\varrho} / S_{\varrho}$ correspond to the choices $\alpha=-\frac{1}{2}$ and $\alpha=\frac{1}{2}$ respectively.

\subsection{CASE $G_{2}$}

Since, for $G_{2}$ its two simple roots are of different lengths, all four families of $C_{-}, S_{-}, S^{s}$ and $S^{l_{-}}$ functions are obtained [15, 16, 25]. The symmetric and antisymmetric orbit functions are given by the following formulas for $a=a_{1} \omega_{1}+a_{2} \omega_{2}$ and $b=b_{1} \alpha_{1}^{\vee}+b_{2} \alpha_{2}^{\vee}$,

$$
\begin{aligned}
C_{a}(b) & =\frac{2}{\left|\operatorname{Stab}_{W} a\right|}\left(\cos 2 \pi\left(a_{1} b_{1}+a_{2} b_{2}\right)\right. \\
& +\cos 2 \pi\left(-a_{1} b_{1}+\left(3 a_{1}+a_{2}\right) b_{2}\right) \\
& +\cos 2 \pi\left(\left(a_{1}+a_{2}\right) b_{1}-a_{2} b_{2}\right) \\
& +\cos 2 \pi\left(\left(2 a_{1}+a_{2}\right) b_{1}-\left(3 a_{1}+a_{2}\right) b_{2}\right) \\
& +\cos 2 \pi\left(\left(-a_{1}-a_{2}\right) b_{1}+\left(3 a_{1}+2 a_{2}\right) b_{2}\right) \\
& \left.+\cos 2 \pi\left(\left(-2 a_{1}-a_{2}\right) b_{1}+\left(3 a_{1}+2 a_{2}\right) b_{2}\right)\right), \\
S_{a}(b) & =2\left(\cos 2 \pi\left(a_{1} b_{1}+a_{2} b_{2}\right)\right. \\
& -\cos 2 \pi\left(-a_{1} b_{1}+\left(3 a_{1}+a_{2}\right) b_{2}\right) \\
& -\cos 2 \pi\left(\left(a_{1}+a_{2}\right) b_{1}-a_{2} b_{2}\right) \\
& +\cos 2 \pi\left(\left(2 a_{1}+a_{2}\right) b_{1}-\left(3 a_{1}+a_{2}\right) b_{2}\right) \\
& +\cos 2 \pi\left(\left(-a_{1}-a_{2}\right) b_{1}+\left(3 a_{1}+2 a_{2}\right) b_{2}\right) \\
& \left.-\cos 2 \pi\left(\left(-2 a_{1}-a_{2}\right) b_{1}+\left(3 a_{1}+2 a_{2}\right) b_{2}\right)\right) .
\end{aligned}
$$

The hybrid cases can be expressed as

$$
\begin{aligned}
S_{a}^{s}(b) & =\frac{2 i}{\left|\operatorname{Stab}_{W} a\right|}\left(\sin 2 \pi\left(a_{1} b_{1}+a_{2} b_{2}\right)\right. \\
& +\sin 2 \pi\left(-a_{1} b_{1}+\left(3 a_{1}+a_{2}\right) b_{2}\right) \\
& -\sin 2 \pi\left(\left(a_{1}+a_{2}\right) b_{1}-a_{2} b_{2}\right) \\
& -\sin 2 \pi\left(\left(2 a_{1}+a_{2}\right) b_{1}-\left(3 a_{1}+a_{2}\right) b_{2}\right) \\
& -\sin 2 \pi\left(\left(-a_{1}-a_{2}\right) b_{1}+\left(3 a_{1}+2 a_{2}\right) b_{2}\right) \\
& \left.-\sin 2 \pi\left(\left(-2 a_{1}-a_{2}\right) b_{1}+\left(3 a_{1}+2 a_{2}\right) b_{2}\right)\right), \\
S_{a}^{l}(b) & =2\left(\sin 2 \pi\left(a_{1} b_{1}+a_{2} b_{2}\right)\right. \\
& -\sin 2 \pi\left(-a_{1} b_{1}+\left(3 a_{1}+a_{2}\right) b_{2}\right) \\
& +\sin 2 \pi\left(\left(a_{1}+a_{2}\right) b_{1}-a_{2} b_{2}\right) \\
& -\sin 2 \pi\left(\left(2 a_{1}+a_{2}\right) b_{1}-\left(3 a_{1}+a_{2}\right) b_{2}\right) \\
& -\sin 2 \pi\left(\left(-a_{1}-a_{2}\right) b_{1}+\left(3 a_{1}+2 a_{2}\right) b_{2}\right) \\
& \left.+\sin 2 \pi\left(\left(-2 a_{1}-a_{2}\right) b_{1}+\left(3 a_{1}+2 a_{2}\right) b_{2}\right)\right) .
\end{aligned}
$$

These functions have been studied in 22, under the notation $C C_{k}, S S_{k}, S C_{k}$ and $C S_{k}$ with

$$
\begin{aligned}
C C_{k}(t) & =\frac{1}{3}\left(\cos \frac{\pi\left(k_{1}-k_{3}\right)\left(t_{1}-t_{3}\right)}{3} \cos \pi k_{2} t_{2}\right. \\
& +\cos \frac{\pi\left(k_{1}-k_{3}\right)\left(t_{2}-t_{1}\right)}{3} \cos \pi k_{2} t_{3} \\
& \left.+\cos \frac{\pi\left(k_{1}-k_{3}\right)\left(t_{3}-t_{2}\right)}{3} \cos \pi k_{2} t_{1}\right) \\
S S_{k}(t) & =\frac{1}{3}\left(\sin \frac{\pi\left(k_{1}-k_{3}\right)\left(t_{1}-t_{3}\right)}{3} \sin \pi k_{2} t_{2}\right. \\
& +\sin \frac{\pi\left(k_{1}-k_{3}\right)\left(t_{2}-t_{1}\right)}{3} \sin \pi k_{2} t_{3} \\
& \left.+\sin \frac{\pi\left(k_{1}-k_{3}\right)\left(t_{3}-t_{2}\right)}{3} \sin \pi k_{2} t_{1}\right)
\end{aligned}
$$




$$
\begin{aligned}
S C_{k}(t) & =\frac{1}{3}\left(\sin \frac{\pi\left(k_{1}-k_{3}\right)\left(t_{1}-t_{3}\right)}{3} \cos \pi k_{2} t_{2}\right. \\
& +\sin \frac{\pi\left(k_{1}-k_{3}\right)\left(t_{2}-t_{1}\right)}{3} \cos \pi k_{2} t_{3} \\
& \left.+\sin \frac{\pi\left(k_{1}-k_{3}\right)\left(t_{3}-t_{2}\right)}{3} \cos \pi k_{2} t_{1}\right), \\
C S_{k}(t) & =\frac{1}{3}\left(\cos \frac{\pi\left(k_{1}-k_{3}\right)\left(t_{1}-t_{3}\right)}{3} \sin \pi k_{2} t_{2}\right. \\
& +\cos \frac{\pi\left(k_{1}-k_{3}\right)\left(t_{2}-t_{1}\right)}{3} \sin \pi k_{2} t_{3} \\
& \left.+\cos \frac{\pi\left(k_{1}-k_{3}\right)\left(t_{3}-t_{2}\right)}{3} \sin \pi k_{2} t_{1}\right),
\end{aligned}
$$

where the variable

$$
t=\left(t_{1}, t_{2}, t_{3}\right) \in \mathbb{R}_{H}^{3}=\left\{t \in \mathbb{R}^{3} \mid t_{1}+t_{2}+t_{3}=0\right\}
$$

and parameter $k=\left(k_{1}, k_{2}, k_{3}\right) \in \mathbb{Z}^{3} \cap \mathbb{R}_{H}^{3}$. Indeed, performing the following change of variables and parameters,

$$
\begin{gathered}
t_{1}=-b_{1}+3 b_{2}, \quad t_{2}=2 b_{1}-3 b_{2}, \quad t_{3}=-b_{1}, \\
k_{1}=a_{1}+a_{2}, \quad k_{2}=a_{1}, \quad k_{3}=-2 a_{1}-a_{2},
\end{gathered}
$$

we obtain the following relations.

$$
\begin{aligned}
& C_{a}=\frac{12}{\left|\operatorname{Stab}_{W} a\right|} C C_{k}, \quad S_{a}=-12 S S_{k}, \\
& S_{a}^{s}=\frac{12 i}{\left|\operatorname{Stab}_{W} a\right|} S C_{k}, \quad S_{a}^{l}=\frac{12 i}{\left|\operatorname{Stab}_{W} a\right|} C S_{k} .
\end{aligned}
$$

In [22], the functions $C_{a}, S_{a+\varrho} / S_{\varrho}, S_{a+\varrho^{s}}^{s} / S_{\varrho^{s}}^{s}$ and $S_{a+\varrho^{l}}^{l} / S_{\varrho^{l}}^{l}$ are expressed as two-variable polynomials in variables

$$
\begin{aligned}
x=\frac{1}{6} C_{\omega_{2}}=\frac{1}{3}\left(\cos 2 \pi b_{2}+\right. & \cos 2 \pi\left(b_{1}-b_{2}\right) \\
& \left.+\cos 2 \pi\left(-b_{1}+2 b_{2}\right)\right), \\
y=\frac{1}{6} C_{\omega_{1}}=\frac{1}{3}\left(\cos 2 \pi b_{1}+\right. & \cos 2 \pi\left(-b_{1}+3 b_{2}\right) \\
& \left.+\cos 2 \pi\left(2 b_{1}-3 b_{2}\right)\right)
\end{aligned}
$$

and it is shown that they are orthogonal within each family with respect to a weighted integral on the region (see Fig. 2 containing points $(x, y)$ satisfying

$$
\left(1+2 y-3 x^{2}\right)\left(24 x^{3}-y^{2}-12 x y-6 x-4 y-1\right) \geq 0
$$

with the weight function $w_{\alpha, \beta}(x, y)$ equal to

$$
\left(1+2 y-3 x^{2}\right)^{\alpha}\left(24 x^{3}-y^{2}-12 x y-6 x-4 y-1\right)^{\beta}
$$

with parameters

$$
\begin{array}{ll}
\alpha=\beta=-\frac{1}{2} & \text { for } C \text {-functions, } \\
\alpha=\beta=\frac{1}{2} & \text { for } S \text {-functions, } \\
\alpha=\frac{1}{2}, \beta=-\frac{1}{2} & \text { for } S^{s} \text {-functions, } \\
\alpha=-\frac{1}{2}, \beta=\frac{1}{2} & \text { for } S^{l} \text {-functions. }
\end{array}
$$

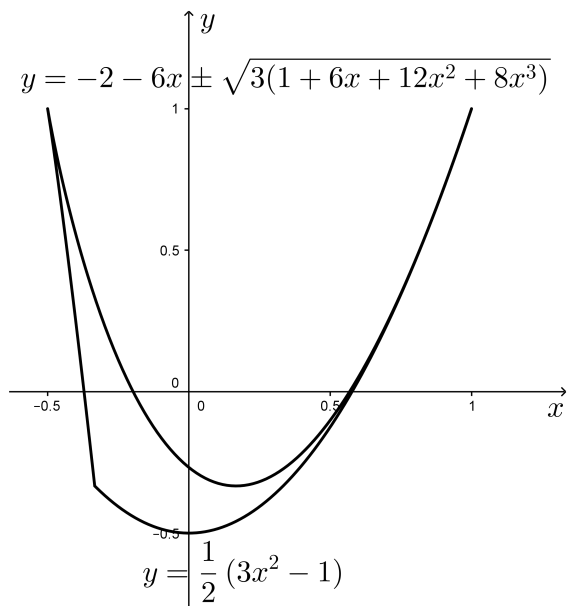

Figure 2. The region of orthogonality for the case $G_{2}$.

\subsection{CASES $B_{n}$ AND $C_{n}$}

It is shown in this section that the $C_{-}, S_{-}, S^{s_{-}}$ and $S^{l}$-functions arising from $B_{n}$ and $C_{n}$ are related to the symmetric and antisymmetric multivariate generalizations of trigonometric functions [17]. The symmetric cosine functions $\cos _{\lambda}^{+}(x)$ and the antisymmetric cosine functions $\cos _{\lambda}^{-}(x)$ of the variable $x=\left(x_{1}, \ldots, x_{n}\right) \in \mathbb{R}^{n}$ are labelled by the parameter $\lambda=\left(\lambda_{1}, \ldots, \lambda_{n}\right) \in \mathbb{R}^{n}$ and are given by the following explicit formulas,

$$
\begin{aligned}
& \cos _{\lambda}^{+}(x) \equiv \sum_{\sigma \in S_{n}} \prod_{k=1}^{n} \cos \left(\pi \lambda_{\sigma(k)} x_{k}\right), \\
& \cos _{\lambda}^{-}(x) \equiv \sum_{\sigma \in S_{n}} \operatorname{sgn}(\sigma) \prod_{k=1}^{n} \cos \left(\pi \lambda_{\sigma(k)} x_{k}\right),
\end{aligned}
$$

where $S_{n}$ denotes the symmetric group consisting of all permutations of numbers $1, \ldots, n$, and $\operatorname{sgn}(\sigma)$ is the signature of $\sigma$. The symmetric sine functions $\sin _{\lambda}^{+}(x)$ and the antisymmetric sine functions $\sin _{\lambda}^{-}(x)$ are defined similarly,

$$
\begin{aligned}
& \sin _{\lambda}^{+}(x) \equiv \sum_{\sigma \in S_{n}} \prod_{k=1}^{n} \sin \left(\pi \lambda_{\sigma(k)} x_{k}\right), \\
& \sin _{\lambda}^{-}(x) \equiv \sum_{\sigma \in S_{n}} \operatorname{sgn}(\sigma) \prod_{k=1}^{n} \sin \left(\pi \lambda_{\sigma(k)} x_{k}\right) .
\end{aligned}
$$

Firstly, consider the Lie algebra $B_{n}$ and an orthonormal basis $\left\{e_{1}, \ldots, e_{n}\right\}$ of $\mathbb{R}^{n}$ such that

$$
\alpha_{i}=e_{i}-e_{i+1} \text { for } i=1, \ldots, n-1 \text { and } \alpha_{n}=e_{n} \text {. }
$$

If we determine any $a \in \mathbb{R}^{n}$ by its coordinates with respect to the basis $\left\{e_{1}, \ldots, e_{n}\right\}$,

$$
a=\left(a_{1}, \ldots, a_{n}\right)=a_{1} e_{1}+\cdots+a_{n} e_{n},
$$

then it holds for the generators $r_{i}, i=1, \ldots, n-1$ and $r_{n}$ of the Weyl group $W\left(B_{n}\right)$ of $B_{n}$ that

$$
\begin{gathered}
r_{i}\left(a_{1}, \ldots, a_{i}, a_{i+1}, \ldots, a_{n}\right)=\left(a_{1}, \ldots, a_{i+1}, a_{i}, \ldots, a_{n}\right), \\
r_{n}\left(a_{1}, \ldots, a_{n-1}, a_{n}\right)=\left(a_{1}, \ldots, a_{n-1},-a_{n}\right) .
\end{gathered}
$$


Therefore, $W\left(B_{n}\right)$ consists of all permutations of the coordinates $a_{i}$ with possible sign alternations of some of them, and we actually have that $W\left(B_{n}\right)$ is isomorphic to $(\mathbb{Z} / 2 \mathbb{Z})^{n} \rtimes S_{n}[12$. This implies that

$$
\begin{aligned}
\Phi_{a}(b)= & \sum_{w \in W\left(B_{n}\right)} e^{2 \pi i\langle w(a), b\rangle} \\
& =\sum_{\sigma \in S_{n}} \prod_{k=1}^{n} \sum_{l_{k}= \pm 1} e^{2 \pi i\left(l_{k} a_{\sigma(k)} b_{k}\right)} \\
= & \sum_{\sigma \in S_{n}} \prod_{k=1}^{n}\left(e^{2 \pi i a_{\sigma(k)} b_{k}}+e^{-2 \pi i a_{\sigma(k)} b_{k}}\right) \\
= & 2^{n} \sum_{\sigma \in S_{n}} \prod_{k=1}^{n} \cos \left(2 \pi a_{\sigma(k)} b_{k}\right)=2^{n} \cos _{a}^{+}(2 b) .
\end{aligned}
$$

Since det is a homomorphism on $W\left(B_{n}\right)$, we also obtain

$$
\begin{aligned}
\varphi_{a}(b) & =\sum_{w \in W\left(B_{n}\right)} \operatorname{det}(w) e^{2 \pi i\langle w(a), b\rangle} \\
& =\sum_{\sigma \in S_{n}} \operatorname{det}(\sigma) \prod_{k=1}^{n} \sum_{l_{k}= \pm 1} l_{k} e^{2 \pi i\left(l_{k} a_{\sigma(k)} b_{k}\right)} \\
= & \sum_{\sigma \in S_{n}} \operatorname{det}(\sigma) \prod_{k=1}^{n}\left(e^{2 \pi i a_{\sigma(k)} b_{k}}-e^{-2 \pi i a_{\sigma(k)} b_{k}}\right) \\
=(2 i)^{n} & \sum_{\sigma \in S_{n}} \operatorname{det}(\sigma) \prod_{k=1}^{n} \sin \left(2 \pi a_{\sigma(k)} b_{k}\right) \\
= & (2 i)^{n} \sin _{a}^{-}(2 b) .
\end{aligned}
$$

Similar connections are valid for $S^{s}$-functions and $S^{l}$-functions,

$$
\varphi_{a}^{s}(b)=(2 i)^{n} \sin _{a}^{+}(2 b), \quad \varphi_{a}^{l}(b)=2^{n} \cos _{a}^{-}(2 b) .
$$

Since Lie algebras $B_{n}$ and $C_{n}$ are dual to each other, we can deduce that the symmetric and antisymmetric generalizations are also connected to the Weyl-orbit functions of $C_{n}$. In order to obtain explicit relations, one can proceed by analogy with case $B_{n}$ and introduce an orthogonal basis $\left\{f_{1}, \ldots, f_{n}\right\}$ such that for $i=1, \ldots, n-1$

$$
\left\langle f_{i}, f_{i}\right\rangle=\frac{1}{2}, \quad \alpha_{i}=f_{i}-f_{i+1} \quad \text { and } \quad \alpha_{n}=2 f_{n} .
$$

We denote by $\tilde{a}_{i}$ the coordinates of any point $a \in$ $\mathbb{R}^{n}$ with respect to the basis $\left\{f_{1}, \ldots, f_{n}\right\}$, i.e. $a=$ $\left(\tilde{a}_{1}, \ldots, \tilde{a}_{n}\right)=\tilde{a}_{1} f_{1}+\cdots+\tilde{a}_{n} f_{n}$. The generators $r_{i}, i=1, \ldots, n-1$ and $r_{n}$ of the Weyl group $W\left(C_{n}\right)$ corresponding to $C_{n}$ are also given by

$$
\begin{gathered}
r_{i}\left(\tilde{a}_{1}, \ldots, \tilde{a}_{i}, \tilde{a}_{i+1}, \ldots, \tilde{a}_{n}\right)=\left(\tilde{a}_{1}, \ldots, \tilde{a}_{i+1}, \tilde{a}_{i}, \ldots, \tilde{a}_{n}\right), \\
r_{n}\left(\tilde{a}_{1}, \ldots, \tilde{a}_{n-1}, \tilde{a}_{n}\right)=\left(\tilde{a}_{1}, \ldots, \tilde{a}_{n-1},-\tilde{a}_{n}\right) .
\end{gathered}
$$

Thus, proceeding as before, we derive the following.

$$
\begin{array}{ll}
\Phi_{a}(b)=2^{n} \cos _{a}^{+}(b), & \varphi_{a}(b)=(2 i)^{n} \sin _{a}^{-}(b), \\
\varphi_{a}^{s}(b)=2^{n} \cos _{a}^{-}(b), & \varphi_{a}^{l}(b)=(2 i)^{n} \sin _{a}^{+}(b) .
\end{array}
$$

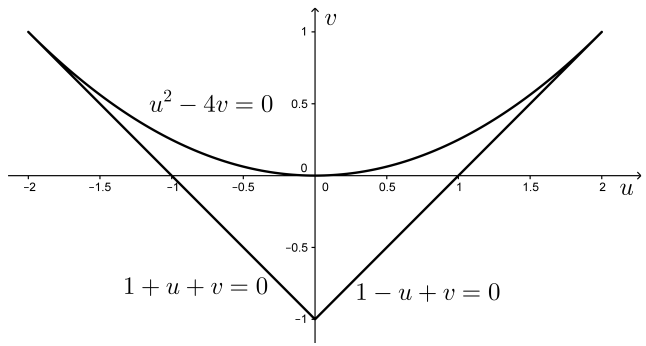

FiguRE 3 . The region of orthogonality bounded by two lines and parabola.

Note that the $S^{s}$-functions are related to $\cos _{a}^{-}$and the $S^{l}$-functions are related to $\sin _{a}^{+}$in the case of $C_{n}$, whereas the $S^{s}$-functions correspond to $\sin _{a}^{+}$and the $S^{l}$-functions correspond to $\cos _{a}^{-}$if we consider the simple Lie algebra $B_{n}$. This follows from the fact that the short (long) roots of $C_{n}$ are dual to the long (short) roots of $B_{n}$.

Setting $n=2$, the construction of the polynomials

$$
\begin{aligned}
& \mathcal{P}_{\left(k_{1}, k_{2}\right)}^{I,+} \equiv \cos _{\left(k_{1}, k_{2}\right)}^{+}, \quad \mathcal{P}_{\left(k_{1}, k_{2}\right)}^{I,-} \equiv \frac{\cos _{\left(k_{1}+1, k_{2}\right)}^{-}}{\cos _{(1,0)}^{-}}, \\
& \mathcal{P}_{\left(k_{1}, k_{2}\right)}^{I I I,+} \equiv \frac{\cos _{\left(k_{1}+\frac{1}{2}, k_{2}+\frac{1}{2}\right)}^{+}}{\cos _{\left(\frac{1}{2}, \frac{1}{2}\right)}^{+}}, \\
& \mathcal{P}_{\left(k_{1}, k_{2}\right)}^{I I I,-} \equiv \frac{\cos _{\left(k_{1}+\frac{3}{2}, k_{2}+\frac{1}{2}\right)}^{-}}{\cos _{\left(\frac{3}{2}, \frac{1}{2}\right)}^{-}}
\end{aligned}
$$

labelled by $k_{1} \geq k_{2} \geq 0$ and in the variables

$$
\begin{aligned}
& X_{1} \equiv \cos _{(1,0)}^{+}\left(x_{1}, x_{2}\right)=\cos \left(x_{1}\right)+\cos \left(x_{2}\right), \\
& X_{2} \equiv \cos _{(1,1)}^{+}\left(x_{1}, x_{2}\right)=2 \cos \left(x_{1}\right) \cos \left(x_{2}\right)
\end{aligned}
$$

yields special cases of two-variable polynomials built in [19 21. These polynomials are constructed by orthogonalization of monomials $1, u, v, u^{2}, u v, v^{2}, \ldots$ of generic variables $u, v$ with respect to the weight function

$$
(1-u+v)^{\alpha}(1+u+v)^{\beta}\left(u^{2}-4 v\right)^{\gamma}
$$

in the domain bounded by the curves $1-u+v=0$, $1+u+v=0$ and $u^{2}-4 v=0$, see Fig. 3 . The parameters $\alpha, \beta, \gamma$ are required to satisfy the conditions $\alpha, \beta, \gamma>-1, \alpha+\gamma+\frac{3}{2}>0$ and $\beta+\gamma+\frac{3}{2}>0$. The resulting polynomials with the highest term $u^{m-k} v^{k}$ are denoted by $p_{m, k}^{\alpha, \beta, \gamma}(u, v)$, where $m \geq k \geq 0$. The polynomial variables $X_{1}$ and $X_{2}$ are related to the variables $u$ and $v$ of [19 21] by

$$
X_{1}=u, \quad X_{2}=2 v
$$

and it can easily be shown that

- $\mathcal{P}_{\left(k_{1}, k_{2}\right)}^{I,+}$ coincides, up to a constant, with $p_{k_{1}, k_{2}}^{\alpha, \gamma}(u, v)$ for $\alpha=\beta=\gamma=-\frac{1}{2}$,

- $\mathcal{P}_{\left(k_{1}, k_{2}\right)}^{I I I,+}$ coincides, up to a constant, with $p_{k_{1}, k_{2}}^{\alpha, \beta, \gamma}(u, v)$ for $\alpha=\gamma=-\frac{1}{2}$ and $\beta=\frac{1}{2}$, 
- $\mathcal{P}_{\left(k_{1}, k_{2}\right)}^{I,-}$ coincides, up to a constant, with $p_{k_{1}, k_{2}}^{\alpha, \beta, \gamma}(u, v)$ for $\alpha=\beta=-\frac{1}{2}$ and $\gamma=\frac{1}{2}$,

- $\mathcal{P}_{\left(k_{1}, k_{2}\right)}^{I I I,-}$ coincides, up to a constant, with $p_{k_{1}, k_{2}}^{\alpha, \beta, \gamma}(u, v)$ for $\alpha=-\frac{1}{2}$ and $\beta=\gamma=\frac{1}{2}$.

\section{Concluding Remarks}

(1.) Symmetric and antisymmetric cosine functions can be used to construct multivariate orthogonal polynomials analogous to the Chebyshev polynomials of the first and third kind. The method of construction is based on decomposition of the products of these functions and is fully described in [7]. To build polynomials analogous to the Chebyshev polynomials of the second and fourth kind, it seems that the symmetric and antisymmetric generalizations of sine functions have to be analysed. This hypothesis is supported by the decomposition of the products of two-dimensional sine functions which can be found in [11].

(2.) Another approach to generalization of the multivariate polynomials related to the Weyl-orbit functions stems from the shifted orthogonality of the orbit functions developed in [2]. This generalization encompasses shifts of the points of the sets over which the functions are discretely orthogonal, and also shifts of the labeling weights. As a special case it contains for $A_{1}$ all four kinds of Chebyshev polynomials. The existence of analogous polynomials obtained through this approach and their relations to already known generalizations deserves further study.

(3.) Besides the methods of polynomial interpolation and numerical integration, the Chebyshev polynomials are connected to other efficient methods in numerical analysis such as numerical solutions of differential equations, solutions of difference equations, fast transforms and spectral methods. The existence and the form of these methods, connected in a multivariate setting to Weyl-orbit functions, are open problems.

\section{ACKNOWLEDGEMENTS}

The authors gratefully acknowledge the support received for this work from RVO68407700. This work is supported by the European Union through the project Support of Inter-sectoral Mobility and Quality Enhancement of Research Teams at the Czech Technical University in Prague CZ.1.07/2.3.00/30.0034.

\section{REFERENCES}

[1] N. Bourbaki, Groupes et algèbres de Lie, Chapiters IV, V, VI, Hermann, Paris 1968.

[2] T. Czyżycki, J. Hrivnák, Generalized discrete orbit function transforms of affine Weyl groups, J. Math. Phys. 55 (2014), 113508, DOI:10.1063/1.4901230.

[3] C. F. Dunkl, Y. Xu, Orthogonal polynomials of several variables, Cambridge University Press, Cambridge, 2001, DOI:10.1017/CBO9781107786134.
[4] A. Erdélyi, W. Magnus, F. Oberhettinger, F. G. Tricomi, Higher transcendental functions. Vol. II, Robert E. Krieger Publishing Co., Inc., Melbourne, Fla., 1981.

[5] D. C. Handscomb, J. C. Mason, Chebyshev polynomials, Chapman\&Hall/CRC, USA, 2003, DOI:10.1201/9781420036114

[6] L. Háková, J. Hrivnák, J. Patera, Four families of Weyl group orbit functions of $B_{3}$ and $C_{3}$, J. Math. Phys. 54 (2013), 083501, 19, DOI:10.1063/1.4817340

[7] J. Hrivnák, L. Motlochová, Discrete transforms and orthogonal polynomials of (anti)symmetric multivariate cosine functions, SIAM J. Numer. Anal. 52 (2014), no. 6, 3021-3055, DOI:10.1137/140964916.

[8] J. Hrivnák, L. Motlochová, J. Patera, On discretization of tori of compact simple Lie groups II., J. Phys. A 45 (2012), 255201, 18, DOI:10.1088/1751-8113/45/25/255201

[9] J. Hrivnák, L. Motlochová, J. Patera, Cubature formulas of multivariate polynomials arising from symmetric orbit functions, Symmetry 8 (2016), no. 7, 63, DOI:10.3390/sym8070063.

[10] J. Hrivnák, J. Patera, On discretization of tori of compact simple Lie groups, J. Phys. A: Math. Theor. 42 (2009), 385208, DOI:10.1088/1751-8113/42/38/385208

[11] J. Hrivnák, L. Motlochová, J. Patera, Two-dimensional symmetric and antisymmetric generalizations of sine functions, J. Math. Phys $\mathbf{5 1}$ (2010), 073509, 13, DOI:10.1063/1.3430567.

[12] J. E. Humphreys, Reflection groups and Coxeter groups, Cambridge Studies in Advanced Mathematics, 29 (1990), Cambridge University Press, Cambridge, DOI:10.1017/CBO9780511623646

[13] J. E. Humphreys, Introduction to Lie algebras and representation theory, Springer-Verlag, New York, 1978, DOI:10.1007/978-1-4612-6398-2

[14] R. Kane, Reflection groups and invariant theory, Springer-Verlag, New York, 2001, DOI:10.1007/978-1-4757-3542-0.

[15] A. U. Klimyk, J. Patera, Orbit functions, SIGMA 2 (2006), 006, 60, DOI:10.3842/SIGMA.2006.006

[16] A. U. Klimyk, J. Patera, Antisymmetric orbit functions, SIGMA 3 (2007), paper 023, 83, DOI:10.3842/SIGMA.2007.023.

[17] A. Klimyk, J. Patera, (Anti)symmetric multivariate trigonometric functions and corresponding Fourier transforms, J. Math. Phys. 48 (2007), 093504, 24, DOI:10.1063/1.2779768

[18] A. W. Knapp, Lie groups beyond an introduction, Birkhäuser Boston Inc., Boston, MA, 1996.

[19] T. H. Koornwinder, Orthogonal polynomials in two variables which are eigenfunctions of two algebraically independent partial differential operators I-II, Kon. Ned. Akad. Wet. Ser. A 77 (1974), 46-66.

[20] T. H. Koornwinder, Orthogonal polynomials in two variables which are eigenfunctions of two algebraically independent partial differential operators III-IV, Indag. Math. 36 (1974), 357-381. 
[21] T. H. Koornwinder, Two-variable analogues of the classical orthogonal polynomials, Theory and

Application of Special Functions, edited by R. A. Askey, Academic Press, New York (1975) 435-495, DOI:10.1016/B978-0-12-064850-4.50015-X

[22] H. Li, J. Sun, Y. Xu, Discrete Fourier Analysis and Chebyshev Polynomials with $G_{2}$ Group, SIGMA 8 (2012), Paper 067, 29, DOI:10.3842/SIGMA.2012.067

[23] H. Li, J. Sun, Y. Xu, Discrete Fourier analysis, cubature and interpolation on a hexagon and a triangle, SIAM J. Numer. Anal. 46 (2008), 1653-1681, DOI:10.1137/060671851

[24] H. Li, Y. Xu, Discrete Fourier analysis on fundamental domain and simplex of $A_{d}$ lattice in d-variables, J. Fourier Anal. Appl. 16, 383-433, (2010), DOI:10.1007/s00041-009-9106-9.

[25] R. V. Moody, L. Motlochová, J. Patera, Gaussian cubature arising from hybrid characters of simple Lie groups, J. Fourier Anal. Appl. 20 (2014), Issue 6, 1257-1290, DOI:10.1007/s00041-014-9355-0

[26] R. V. Moody, J. Patera, Cubature formulae for orthogonal polynomials in terms of elements of finite order of compact simple Lie groups, Advances in Applied Mathematics 47 (2011) 509-535, DOI:10.1016/j.aam.2010.11.005

[27] R. V. Moody and J. Patera, Orthogonality within the families of $C_{-}, S_{-}$, and $E$-functions of any compact semisimple Lie group, SIGMA 2 (2006) 076, 14, DOI:10.3842/SIGMA.2006.076

[28] J. Patera, A. Zaratsyan, Discrete and continuous sine transform generalized to semisimple Lie groups of rank two, J. Math. Phys. 47 (2006), 043512, 22, DOI:10.1063/1.2191361

[29] J. Patera, A. Zaratsyan, Discrete and continuous cosine transform generalized to Lie groups $S U(3)$ and G(2), J. Math. Phys. 46 (2005), 113506, 17, DOI:10.1063/1.2109707

[30] J. Patera, A. Zaratsyan, Discrete and continuous cosine transform generalized to Lie groups $S U(2) \times S U(2)$ and $O(5)$, J. Math. Phys. 46 (2005), 053514, 25, DOI:10.1063/1.1897143

[31] T. J. Rivlin, The Chebyshev polynomials, Wiley, New York, 1974.

[32] J.-P. Serre, Complex semisimple Lie algebras, Springer Monographs in Mathematics, Springer-Verlag, Berlin, 2001, DOI:10.1007/978-3-642-56884-8

[33] G. Szegő, Orthogonal polynomials, American Mathematical Society, Providence, R.I., 1975.

[34] N. Ja. Vilenkin, A. U. Klimyk, Representation of Lie groups and special functions, Kluwer Academic Publishers Group, Dordrecht, 1995, DOI:10.1007/978-94-017-2885-0 